\title{
Leukemia cell proliferation and death in chronic lymphocytic leukemia patients on therapy with the BTK inhibitor ibrutinib
}

\author{
Jan A. Burger, ${ }^{1}$ Kelvin W. Li, ${ }^{2}$ Michael J. Keating, ${ }^{1}$ Mariela Sivina, ${ }^{1}$ Ahmed M. Amer, ${ }^{3}$ Naveen Garg, ${ }^{3}$ \\ Alessandra Ferrajoli, ${ }^{1}$ Xuelin Huang, ${ }^{4}$ Hagop Kantarjian, ${ }^{1}$ William G. Wierda, ${ }^{1}$ Susan O'Brien, ${ }^{1}$ \\ Marc K. Hellerstein, ${ }^{5}$ Scott M. Turner, ${ }^{2}$ Claire L. Emson, ${ }^{2}$ Shih-Shih Chen, ${ }^{6}$ Xiao-Jie Yan, ${ }^{6}$ \\ Dominik Wodarz, ${ }^{7}$ and Nicholas Chiorazzi ${ }^{6}$ \\ 'Department of Leukemia, MD Anderson Cancer Center, Houston, Texas, USA. ${ }^{2}$ KineMed Inc., Emeryville, California, USA. \\ ${ }^{3}$ Department of Diagnostic Radiology and 4Department of Biostatistics, MD Anderson Cancer Center, Houston, Texas, USA \\ ${ }^{5}$ Department of Nutritional Sciences and Toxicology, University of California, Berkeley, Berkeley, California, USA. ${ }^{6}$ Karches \\ Center for Chronic Lymphocytic Leukemia Research, The Feinstein Institute for Medical Research, Northwell Health, \\ Manhasset, New York, USA. 'Department of Mathematics and Department of Ecology and Evolutionary Biology, University \\ of California, Irvine, Irvine, California, USA.
}

BACKGROUND. Ibrutinib is an effective targeted therapy for patients with chronic lymphocytic leukemia (CLL) that inhibits Bruton's tyrosine kinase (BTK), a kinase involved in B cell receptor signaling.

METHODS. We used stable isotopic labeling with deuterated water $\left({ }^{2} \mathrm{H}_{2} \mathrm{O}\right)$ to measure directly the effects of ibrutinib on leukemia cell proliferation and death in $\mathbf{3 0}$ patients with CLL.

RESULTS. The measured average CLL cell proliferation ("birth") rate before ibrutinib therapy was $0.39 \%$ of the clone per day (range $0.17 \%-1.04 \%$ ); this decreased to $0.05 \%$ per day (range $0 \%$ $0.36 \%$ ) with treatment. Death rates of blood CLL cells increased from $0.18 \%$ per day (average, range $0 \%-0.7 \%$ ) prior to treatment to $1.5 \%$ per day (range $0 \%-3.0 \%$ ) during ibrutinib therapy, and they were even higher in tissue compartments.

CONCLUSIONS. This study provides the first direct in vivo measurements to our knowledge of ibrutinib's antileukemia actions, demonstrating profound and immediate inhibition of CLL cell proliferation and promotion of high rates of CLL cell death.

TRIAL REGISTRATION. This trial was registered at clinicaltrials.gov (NCT01752426).

FUNDING. This study was supported by a Cancer Center Support Grant (National Cancer Institute grant P30 CA016672), an NIH grant (CA081554) from the National Cancer Institute, MD Anderson's Moon Shots Program in CLL, and Pharmacyclics, an AbbVie company.

Role of funding source: The study was sponsored, in part, by Pharmacyclics, an AbbVie company.

Conflict of interest: K.W. Li, M.K. Hellerstein, S.M. Turner, and C.L. Emson are employees of or consultants to KineMed Inc. J.A. Burger, N. Chiorazzi, and $S$. O'Brien received research funding from Pharmacyclics, and J.A. Burger received speaking fees from Janssen Pharmaceuticals.

Submitted: August 2, 2016 Accepted: December 13, 2016 Published: January 26, 2017

Reference information: JCI Insight. 2017;2(2):e89904. doi:10.1172/jci.insight.89904.

\section{Introduction}

Treatment of patients with chronic lymphocytic leukemia (CLL) is undergoing fundamental changes $(1,2)$. This is due to the emergence of new therapeutic modalities, such as kinase inhibitors that target critical survival and proliferation signals, especially B cell receptor (BCR) signaling $(1,3,4)$, and to the development of novel monoclonal antibodies directed toward key surface molecules (5).

Ibrutinib (previously called PCI-32765) is a potent $\left(\mathrm{IC}_{50}, 0.5 \mathrm{nM}\right)$, selective inhibitor of Bruton's tyrosine kinase (BTK) that inactivates the enzyme by irreversible covalent bonding to Cys-481 in the ATP-binding domain of BTK $(6,7)$. For patients with CLL, ibrutinib is administered once daily orally at a fixed dose of $420 \mathrm{mg}$ until disease progression or toxicity occurs. Ibrutinib is particularly active in patients with CLL $(1,4,8)$, with overall response rates of $86 \%$ in treatment-naive (TN) patients (9) and $42.6 \%$ in relapsed/refractory (R/R) patients (4), which can increase over time to $90 \%$ with longer follow-up (10). These responses are independent of clinical and genomic risk factors present prior to treatment, including advanced-stage disease, numbers of prior treatments, and the presence of $17 \mathrm{p}$ deletion (1). After 30 months 
on treatment, the estimated progression-free survival rate is $96 \%$ in $\mathrm{TN}$ and $69 \%$ in R/R CLL patients, and the rate of overall survival is $97 \%$ in TN and $79 \%$ in R/R CLL patients.

Clinical responses to ibrutinib are characterized by rapid shrinkage of enlarged lymph nodes and spleen during the first weeks of therapy. This occurs along with a rapid onset and transient increase in peripheral blood leukemia cell counts. This lymphocytosis is variable among patients and relates to the direct presence of the drug. In the first-in-human study using an intermittent dosing schedule, increased absolute lymphocyte counts (ALCs) rapidly dropped during the 7-day ibrutinib-free period, presumably due to rehoming of CLL cells into the tissues, and then increased again once ibrutinib was restarted (8). For most patients, the transient lymphocytosis is asymptomatic and usually resolves during the first 8 months of therapy (11). Although not proven directly in patients in vivo, lymphocytosis is believed to be due to redistribution of CLL cells from tissue compartments into the peripheral blood (12) and not to disease progression (13), i.e., proliferation of CLL cells. Preclinical studies in CLL demonstrated that ibrutinib effectively inhibits BCR signaling, leukemia cell proliferation, survival, migration, and adhesion in vitro (14-16) as well as disease progression in vivo using CLL mouse models $(15,17,18)$. The in vivo mechanisms of action of ibrutinib in CLL patients have not, however, been established.

To study the actions of ibrutinib directly in patients with CLL, we metabolically labeled the DNA of proliferating CLL cells in vivo with deuterium $\left({ }^{2} \mathrm{H}\right)$ by asking patients to drink deuterated "heavy" water $\left({ }^{2} \mathrm{H}_{2} \mathrm{O}\right)$ over a 4-week period before initiating therapy with ibrutinib (Figure 1). Monitoring the rate of production of ${ }^{2} \mathrm{H}$-marked CLL tumor cells over time before therapy and the rate of loss or dilution by unlabeled cells after therapy allowed us to determine the effects of ibrutinib treatment on leukemia cell kinetics (proliferation and death rates) and mobilization of cells from lymphoid tissues (trafficking). In addition, we analyzed volumetric changes in secondary lymphoid organs during ibrutinib therapy, correlating those with changes in peripheral blood CLL cell counts over time, and, using mathematical modeling, calculated CLL cell death rates in blood and tissue separately.

\section{Results}

CLL cell birth and death rates estimated by heavy water $\left({ }^{2} \mathrm{H}_{2} \mathrm{O}\right)$ labeling. The average CLL cell birth rate before ibrutinib therapy was $0.39 \% \pm 0.21 \%$ per day (mean \pm SD, $n=30$ ), based on the appearance rate of labeled cells over the first 8 weeks of the study prior to start of ibrutinib therapy (Figure 2). Birth rates ranged between $0.17 \%$ and $1.04 \%$ per day (Figure 2, B and F), with no detectable difference between unmutated CLL (U-CLL) and mutated CLL (M-CLL) $(0.43 \% \pm 0.20 \%$ per day and $0.43 \pm 0.21 \%$ per day for U-CLL and M-CLL, respectively). After initiating ibrutinib therapy (treatment phase) following washout of ${ }^{2} \mathrm{H}_{2} \mathrm{O}$ from body water, the fraction of ${ }^{2} \mathrm{H}$-labeled CLL cells remained stable for the remainder of the study. The mean fraction of previously divided, ${ }^{2} \mathrm{H}$-labeled cells remained constant at $19.4 \% \pm 0.5 \%$ (Figure $2 \mathrm{C}$ ). Moreover, although there was an initial increase in ALCs in the blood in 26 of 30 patients, the incoming cells were composed of the same proportion of ${ }^{2} \mathrm{H}$-labeled and unlabeled CLL cells as that in circulation (i.e., the fraction of labeled cells did not change despite increases in cell number). Specifically, while wbc and ALCs increased immediately after the start of ibrutinib therapy (Figure 2E and Figure 3, A and B), there was no significant change in the proportion of labeled cells, maintaining a plateau in the fraction of labeled CLL cells (Figure 2C). This observation demonstrates that the additional cells in the bloodstream had not divided after the start of ibrutinib therapy, as any newly divided cells would not be ${ }^{2} \mathrm{H}$ labeled. Furthermore, during the subsequent treatment phase, the number of peripheral blood CLL cells declined progressively, but the proportion of circulating ${ }^{2} \mathrm{H}$-labeled CLL cells did not decrease appreciably (Figure 2C), indicating that very few newly divided, unlabeled cells had entered the circulation during the ibrutinib treatment period.

These results are consistent with the conclusion that ibrutinib had a profound inhibitory effect on leukemia cell proliferation. Indeed, the proportion of labeled CLL cells declined at the rate of $0.05 \% \pm 0.09 \%$ per day $(n=30)$ during this time, indicative of the relatively slow rate at which newly divided (and unlabeled) CLL cells were appearing in the circulation. Comparison of the CLL cell birth rate prior to ibrutinib therapy, calculated from the rate of ${ }^{2} \mathrm{H}$ incorporation into newly divided (labeled) CLL cells, with the CLL cell birth rate after initiating ibrutinib therapy, calculated from the rate of dilution of ${ }^{2} \mathrm{H}-1$ abeled CLL cells by newly divided (unlabeled) CLL cells, revealed that the measured birth rate of CLL cells decreased from $0.39 \% \pm 0.21 \%$ per day (mean $\pm \mathrm{SD}, n=30)$ to $0.05 \% \pm 0.09 \%(n=30)$. Although we did not include an untreated group here for the washout phase, it should be noted that the dilution of CLL cells after washout 


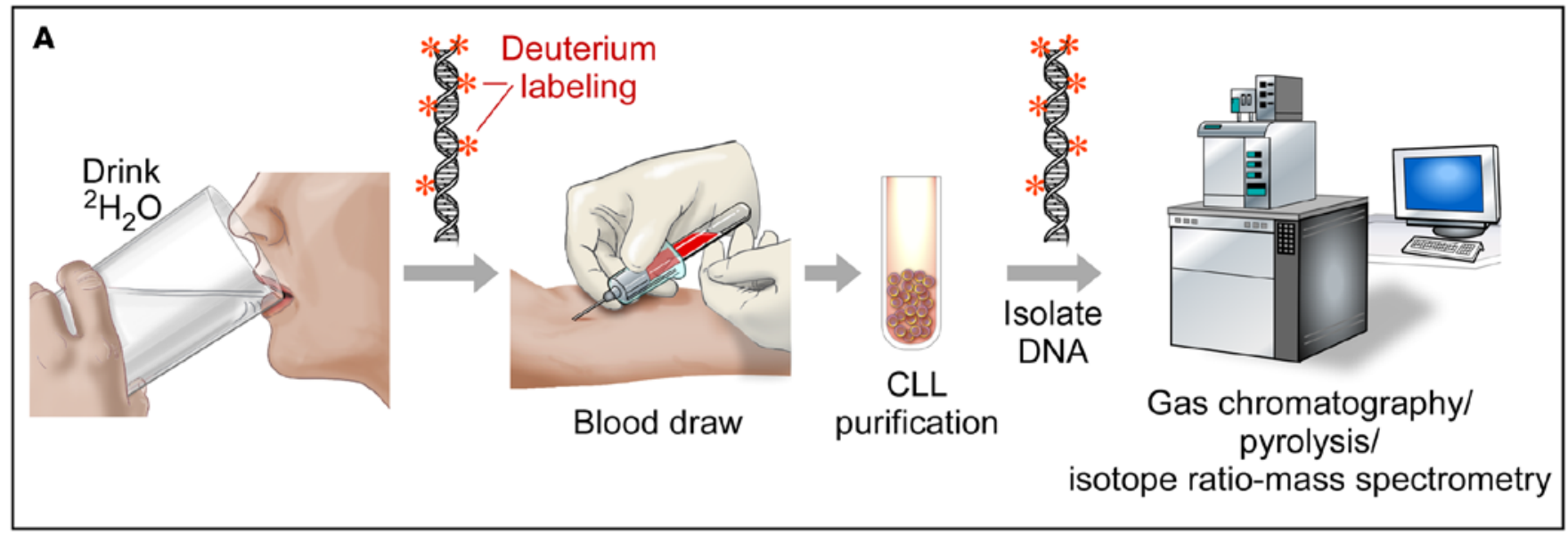

B

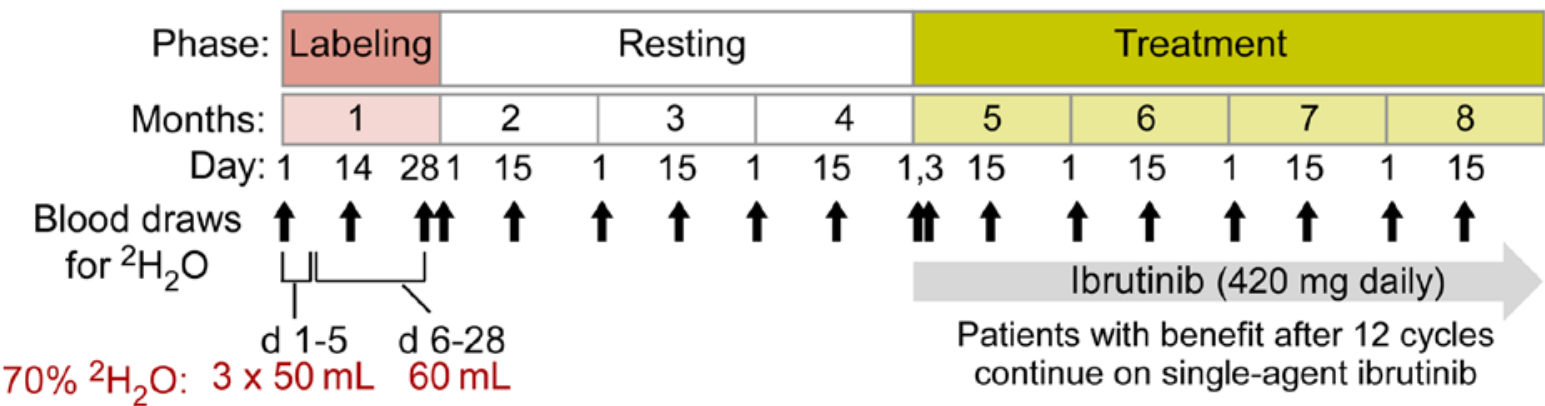

Figure 1. Heavy water labeling in CLL patients before ibrutinib therapy: workflow and trial outline. (A) Workflow diagram to illustrate the principles of the study. Patients ingest heavy water $\left({ }^{2} \mathrm{H}_{2} \mathrm{O}\right)$, and deuterium $\left({ }^{2} \mathrm{H}\right)$ becomes incorporated into the DNA of dividing cells, including dividing CLL cells. Repeated blood draws and purification of CLL cells, followed by DNA isolation, allows quantification of the fraction of labeled DNA in the CLL cells at each time point via gas chromatography/pyrolysis/isotope ratio-mass spectometry. (B) The three phases of the trial - labeling, resting, and treatment - and the duration of each are indicated on the horizontal axis (months, days); 30 patients participated in all three phases of the trial. Blood samples were drawn every 2 weeks at the days indicated by the arrows. Ibrutinib treatment started after the resting period with 420 mg orally daily. Patients benefiting from ibrutinib were allowed to continue on treatment.

of ${ }^{2} \mathrm{H}_{2} \mathrm{O}$ has previously been monitored in over 100 untreated CLL patients (19, 20). Dilution of ${ }^{2} \mathrm{H}$-labeled CLL cells is measurable and comparable to proliferation rates based on ${ }^{2} \mathrm{H}_{2} \mathrm{O}$ incorporation in untreated subjects, in contrast to the findings here on ibrutinib therapy. These findings demonstrate a profound antiproliferative action of the drug.

The above results indicate clearly that the proliferation of CLL in patients is dramatically reduced by ibrutinib therapy. Furthermore, after initiating ibrutinib therapy, the CLL cell death rate increased from $0.18 \% \pm 0.18 \%$ per day (mean $\pm \mathrm{SD}, n=30$ ) prior to therapy to $1.5 \% \pm 0.83 \%$ per day on ibrutinib treatment ( $n=30$, see Figure $2 \mathrm{~F}$ ); this value for CLL cell death rate ranged from $0.16 \%$ to $3.0 \%$ among patients and did not differ significantly between U-CLL $(1.79 \pm 0.80, n=17)$ and M-CLL patients $(1.32 \pm 0.68, n$ $=11, P=0.13$ ). The fits to ALC were linear from the beginning of the ibrutinib treatment period (Figure $2 \mathrm{E})$, consistent with an immediate and relatively constant effect of treatment. Similarly, the lack of dilution of labeled CLL cells by newly divided unlabeled cells (Figure 2C) was apparent from the start of ibrutinib treatment. These kinetic studies indicate that significant changes are observed in the birth and death rates of CLL cells, which occur rapidly and stably, in patients receiving ibrutinib therapy.

Clinical responses and changes in peripheral blood cell counts and marrow infiltration during therapy. Figure 3 depicts the trend of peripheral blood cell counts, bone marrow infiltration, and progression-free and overall survival during ibrutinib treatment in this cohort of CLL patients. Redistribution lymphocytosis was seen only in patients with mutated IGHV (M-CLL); in contrast, redistribution lymphocytosis was absent patients with unmutated IGHV (U-CLL). When comparing patients with M-CLL with patients with U-CLL, there was faster bone marrow clearance of leukemia cells in patients with U-CLL. Further details about reasons for treatment initiation and the clinical outcome are provided in the supplement as Supple- 
A
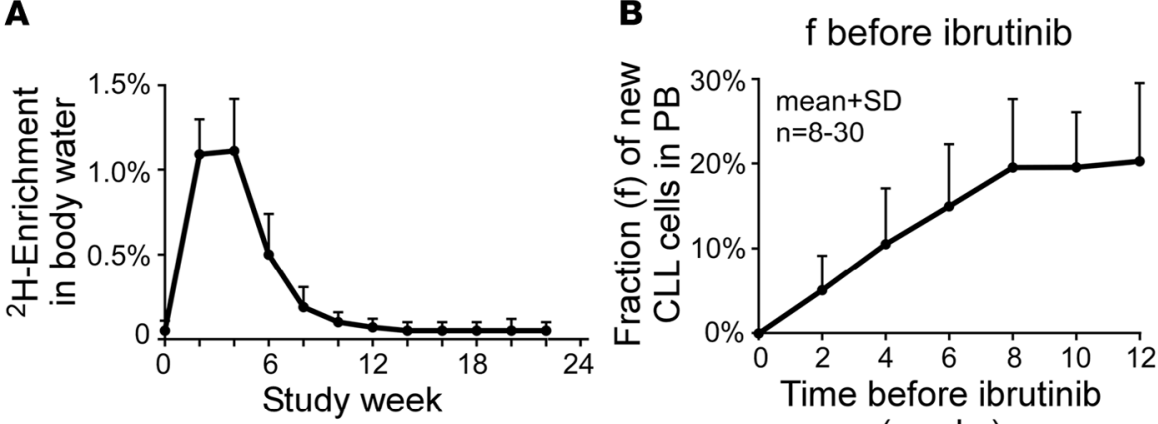

(weeks)

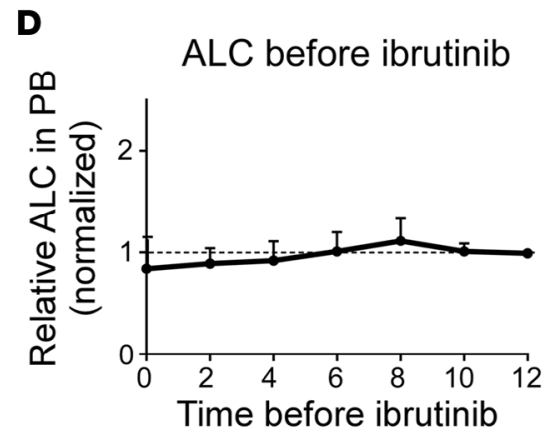

(weeks)

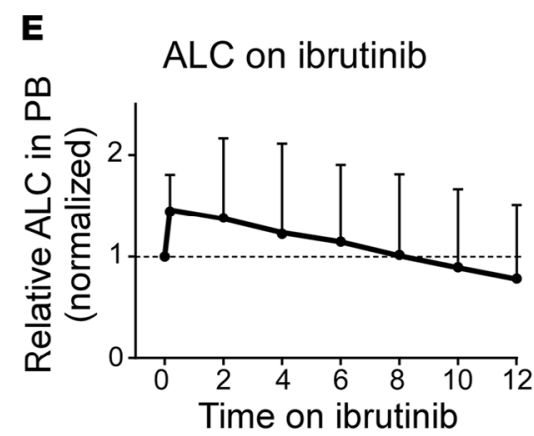

(weeks)

\section{$\mathbf{F}$}

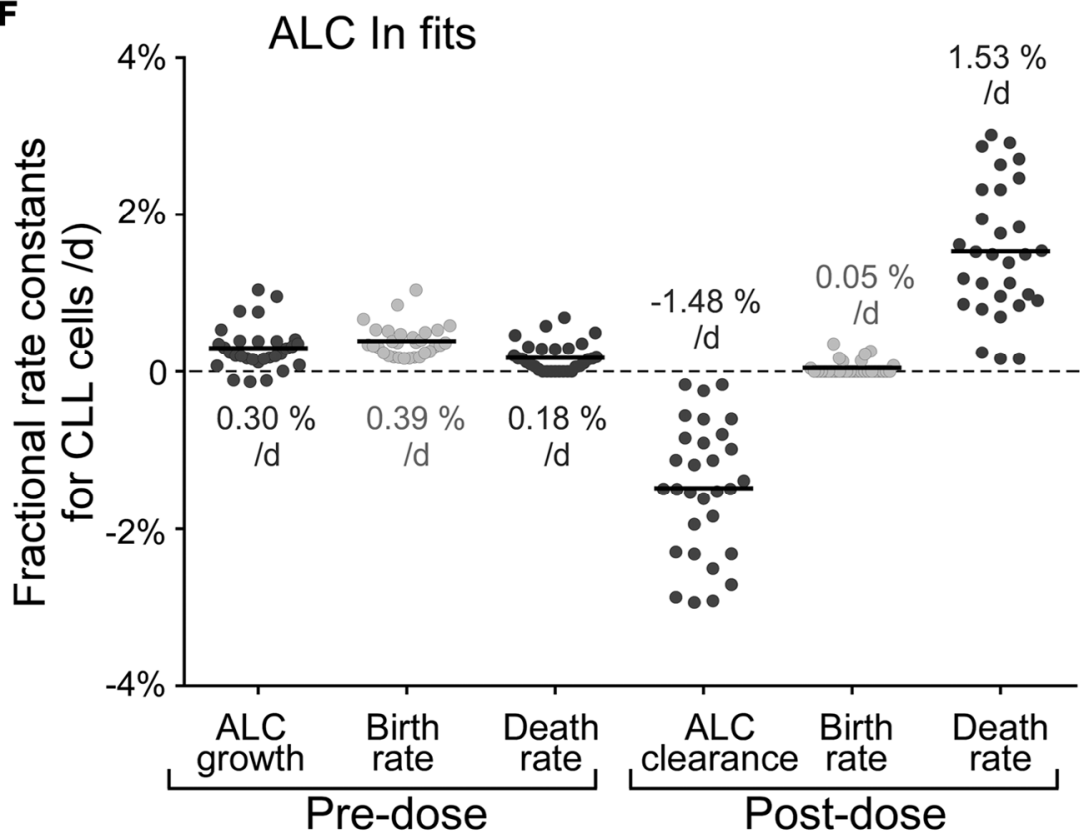

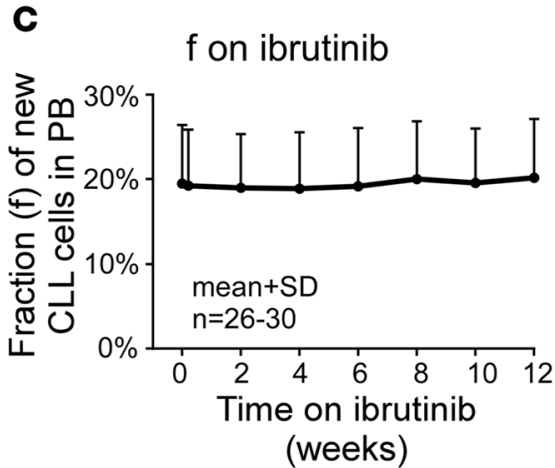

Figure 2. Ibrutinib inhibits CLL cell birth and permits increased CLL cell death. (A) ${ }^{2} \mathrm{H}_{2} \mathrm{O}$ enrichment data from the plasma of CLL patients $(n=30)$, showing enrichment of ${ }^{2} \mathrm{H}_{2} \mathrm{O}$ during the first 4 weeks of ingestion, followed by dilution of ${ }^{2} \mathrm{H}_{2} \mathrm{O}$ during the washout phase, which fits an exponential decay model. (B) Percentage of ${ }^{2} \mathrm{H}$ enrichment in the DNA of peripheral blood CLL cells from the $30 \mathrm{CLL}$ patients studied was measured and converted into a fraction ( $f$ ) of newly divided cells, as described in Methods. Data are plotted for the first 12 weeks of the study, comprising the first 4 weeks of labeling, followed by washout. (C) After starting ibrutinib therapy, there is a plateau in the proportion of labeled CLL cells, indicating an arrest of CLL cell birth based on the absence of dilution in $f$ (the fraction of previously divided, labeled CLL cells) by newly divided, unlabeled CLL cells. (D) The increase in ALC before the start of ibrutinib therapy is due to birth rates that are higher than death rates (see F). (E) After the start of ibrutinib, the ALC initially increases briefly, due to redistribution lymphocytosis and then continuously declines. (F) Calculated birth and death rates before start of ibrutinib therapy (pre-dose) and after the start of ibrutinib therapy (post-dose) demonstrate profound inhibition of CLL proliferation in addition to accelerated CLL cell death and ALC clearance after the start of ibrutinib.

mental Table 1 and in the section called Clinical responses, respectively (supplemental material available online with this article; doi:10.1172/jci.insight.89904DS1).

Tissue CLL cell death rates estimated by computational modeling. Tissue cell death rates, based on changes in tissue volumes over time (Figure 4) and other variables (trending peripheral blood counts, estimated blood volumes), were estimated with mathematical models according to previously established methods (21) (Figure 5, A and B) and correlated with $I G H V$ mutational status. Patients with higher tissue cell death rates more often had unmutated $I G H V$ (U-CLL), while patients with slower disease resolution were more likely to be $I G H V$-mutated (M-CLL) (Figure 5C). The average estimated tissue CLL cell death rate among patients with U-CLL $(28.88 \% \pm 11.33 \%$ per day $)$ was significantly higher than the average death 
A

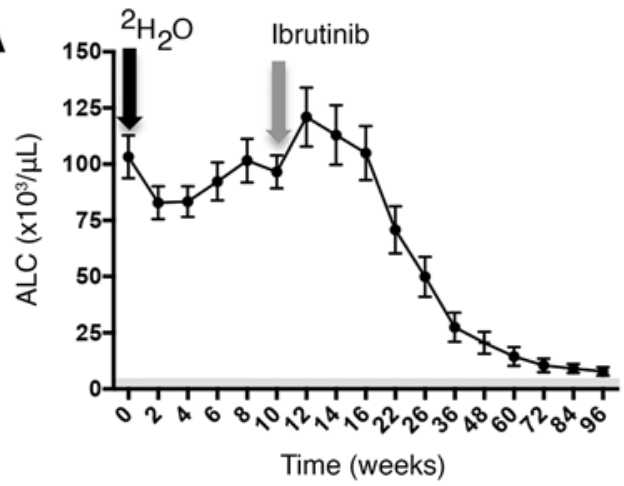

C

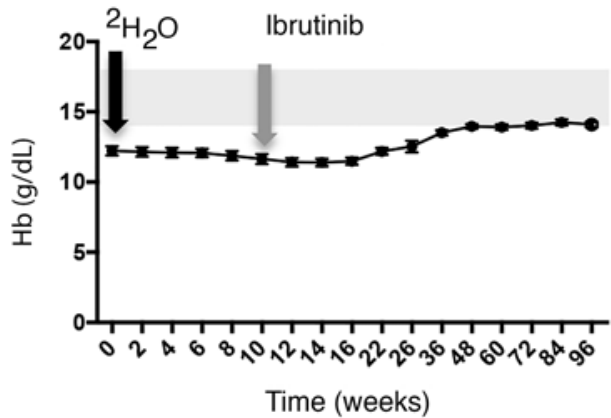

E

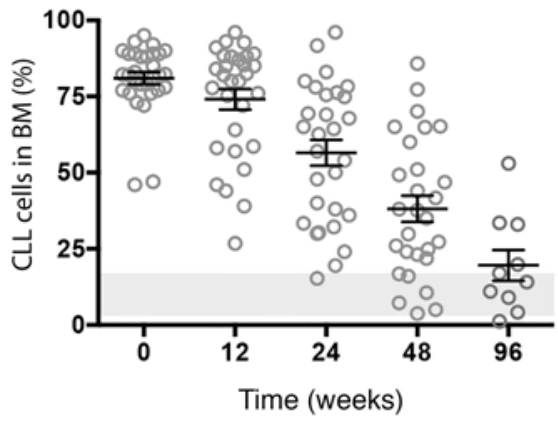

G

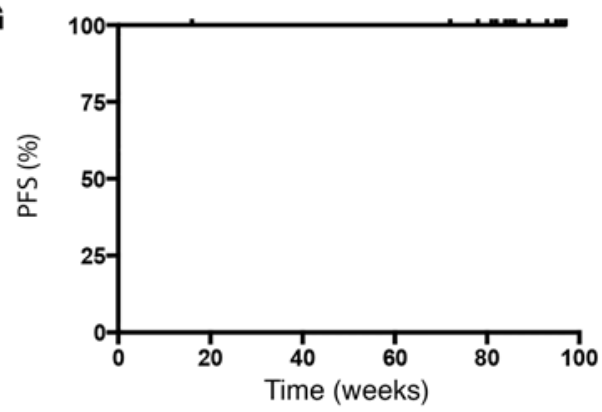

B

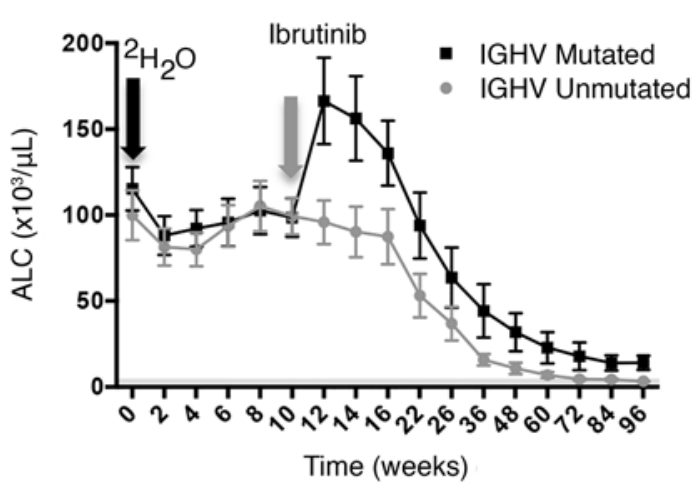

D

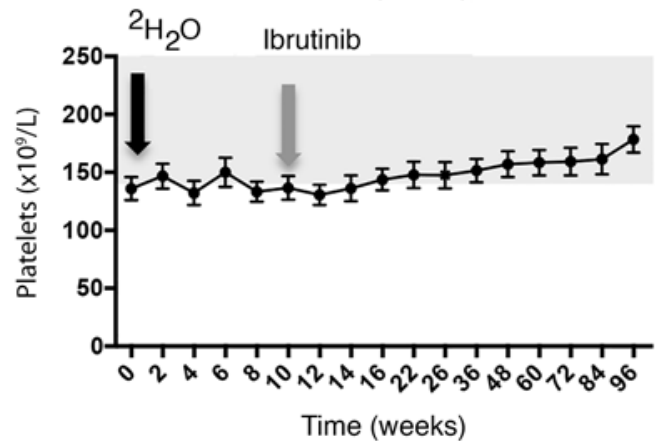

$\mathbf{F}$

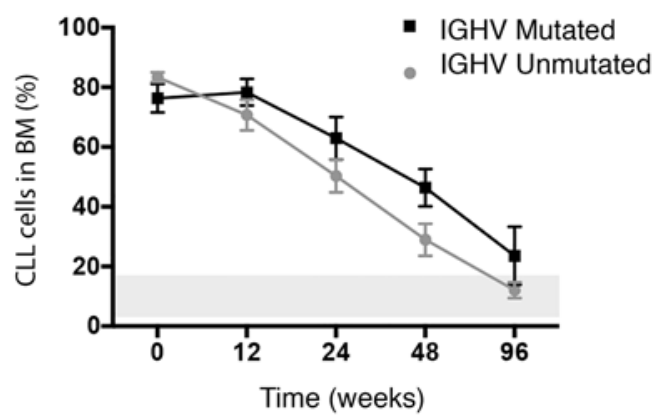

H

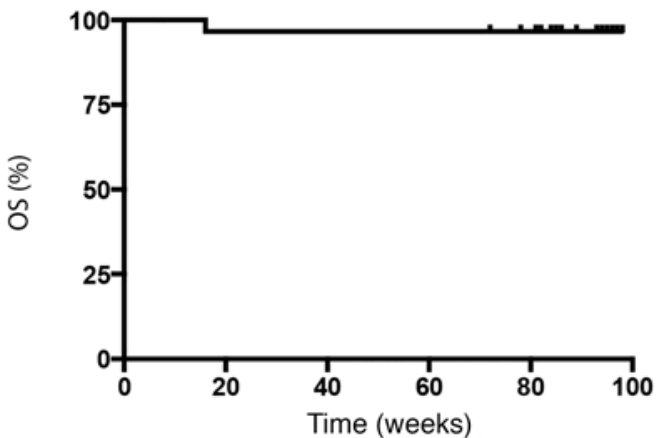

Figure 3. Peripheral blood cell counts, bone marrow infiltration, and survival during ibrutinib treatment. (A) Median ALC ( \pm SEM) before and after starting ibrutinib therapy (black arrow: start of labeling phase; gray arrow: start of ibrutinib therapy). The trend of the ALC shows a characteristic transient increase after the start of ibrutinib therapy due to redistribution of tissue CLL cells into the peripheral blood. (B) Redistribution lymphocytosis was seen only in patients with mutated IGHV (M-CLL, $n=11)$; in contrast, redistribution lymphocytosis was absent patients with unmutated ICHV (U-CLL, $n=17)$. (C) Median hemoglobin levels and (D) platelet counts ( \pm SEM) normalize during ibrutinib therapy. The gray shaded areas indicate normal values. (E) There was a continuous improvement in bone marrow infiltration by CLL cells after starting ibrutinib therapy, indicated as the percentage of bone marrow lymphocytes that are displayed for each patient together with the median $( \pm$ SEM, $n=29$ ). (F) When comparing patients with M-CLL with patients with $U-C L L$, there was faster bone marrow clearance of leukemia cells in patients with U-CLL. (C) No progression events were seen in the entire patient population after a median follow-up of 26 months. (H) Only one CLL- and treatment-unrelated death occurred; the remaining patients are alive and almost all (27 of 30 ) continue on ibrutinib therapy. PFS, progression-free survival; OS, overall survival. 
$\mathbf{A}$
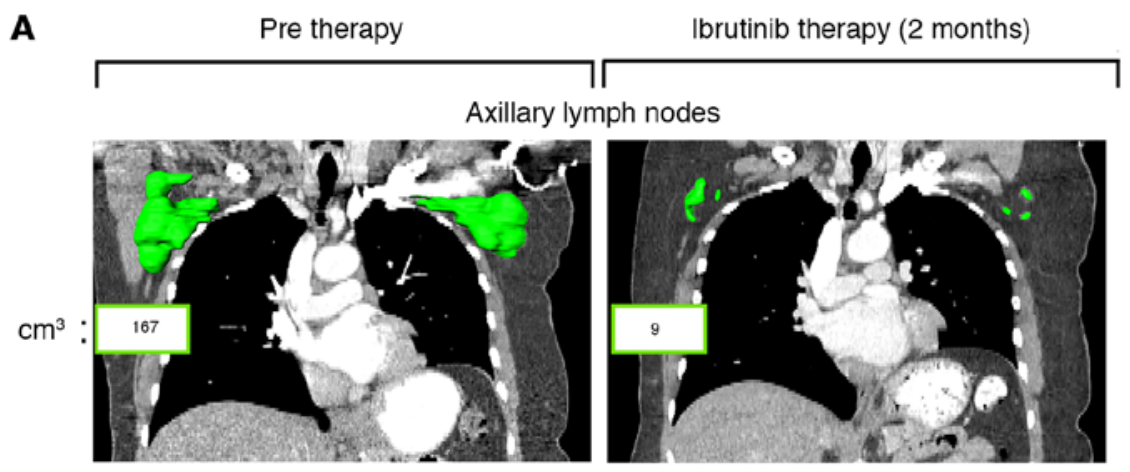

B

Spleen

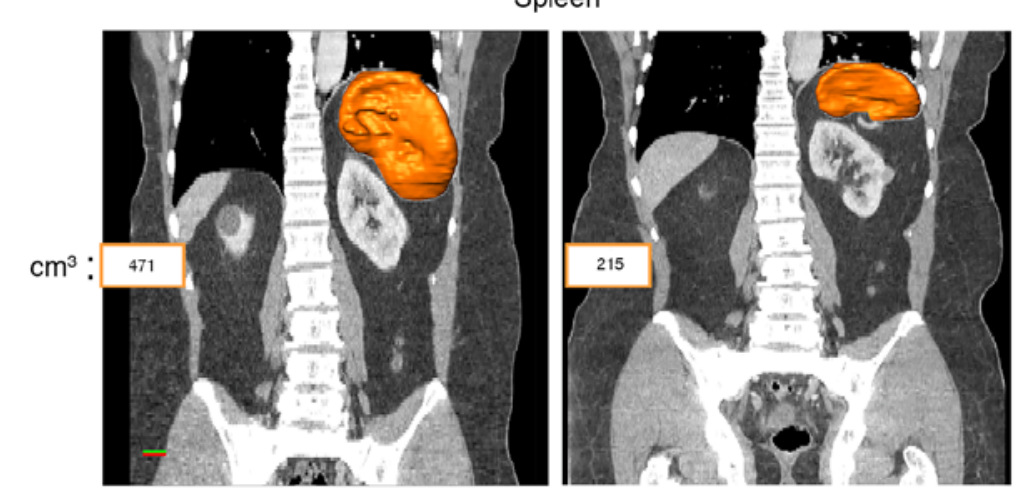

C Abdominal lymph nodes
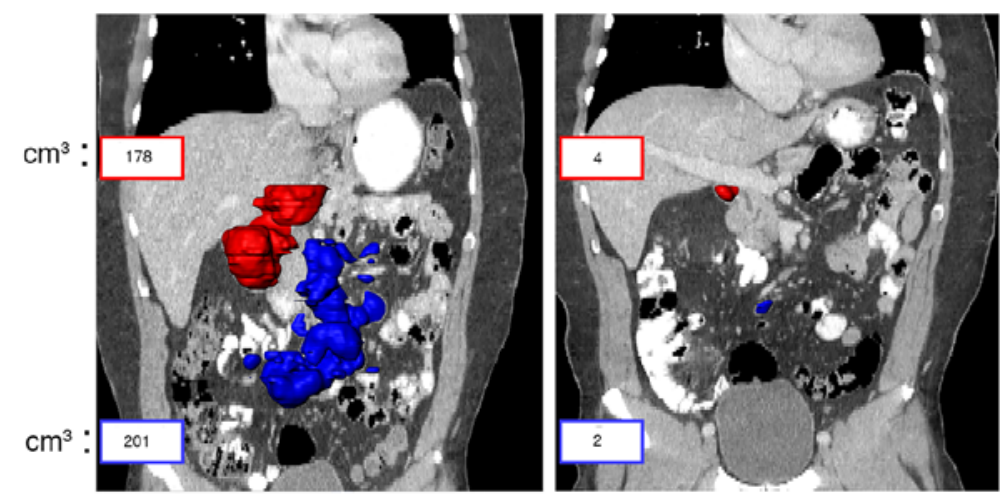

Figure 4. Volumetric analyses of CLL lymph node and spleen manifestation before and after 2 months of therapy with ibrutinib. CT images from a patient with CLL from our series with superimposed reconstruction of main areas of CLL involvement highlighted in color. The volumes (in cubic centimeters) of the axillary (green), mesenteric (blue), other intraabdominal (red), and spleen (orange) disease sites are displayed next to each involved area. (A) Axillary lymph nodes; (B) spleen; and (C) abdominal lymph nodes.

rate among patients with M-CLL $(12.06 \% \pm 9.42 \%$ per day, $P=0.0017)$. This difference is reflected in Figure 5D, which plots the average predicted tissue decline during treatment. Further details about CLL tissue death rate estimates and associated methodologies as well as clinical responses are provided in the supplement in the section called Mathematical modeling and estimation of tissue death rates section as well as the section called Clinical responses.

\section{Discussion}

The data presented here demonstrate directly in vivo in CLL patients that ibrutinib has immediate and profound effects on CLL cell proliferation and death and that the initial lymphocytosis is not due to CLL cell proliferation. We noted robust, immediate inhibition of CLL cell birth, which decreased from 
A

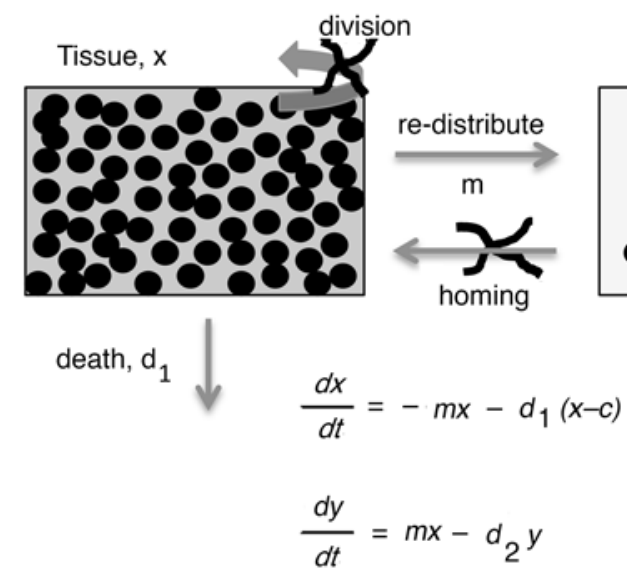

C

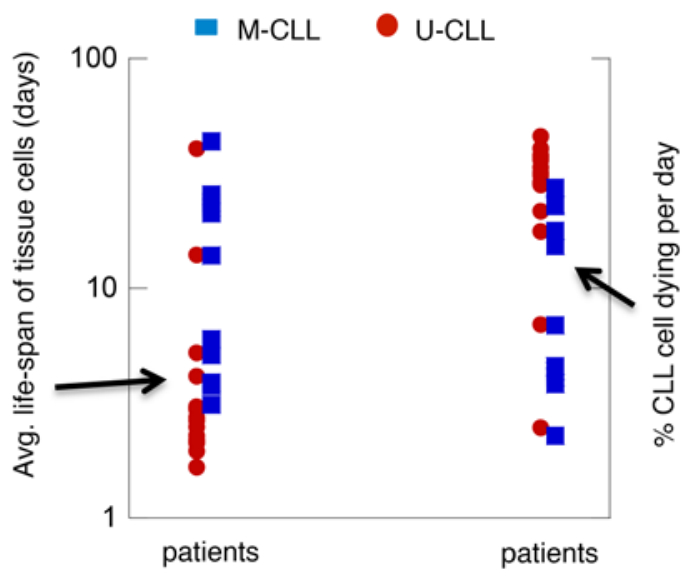

B

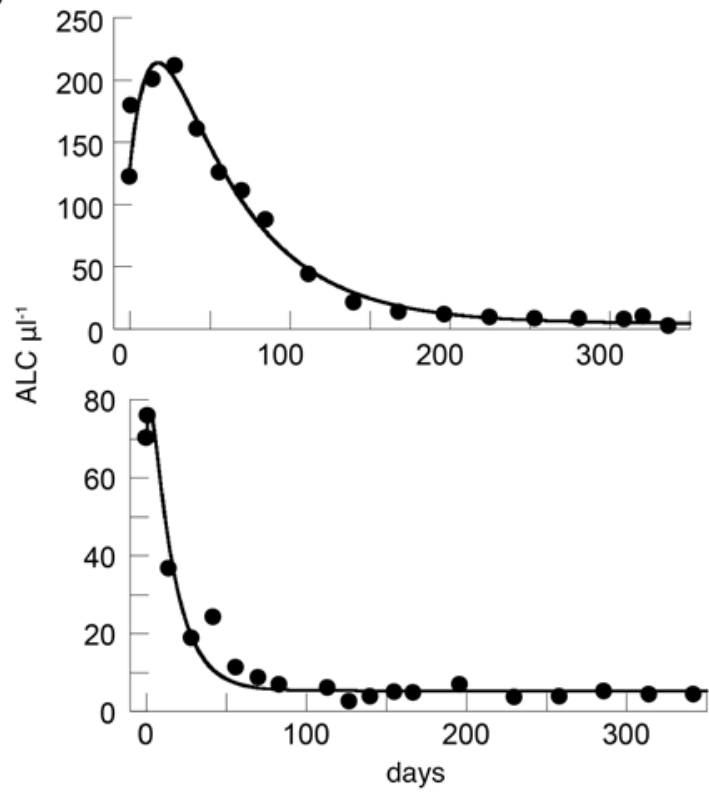

D

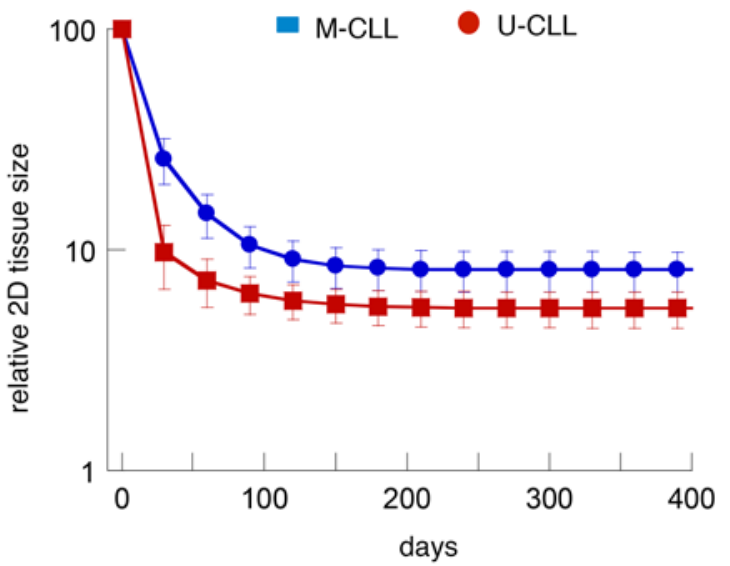

Figure 5. Modeling and estimating CLL kinetics. (A) A 2-compartment mathematical model (given by ordinary differential equations) was used to estimate CLL cell death rates in tissue and blood, according to previously established methods. CLL cells in the tissue are assumed to die with a rate $d_{1}$ and redistribute to blood with a rate $\mathrm{m}$. CLL cells in the blood are assumed to die with a rate $\mathrm{d}_{2}$. The parameter $\mathrm{c}$ is included to account for the observation that the rate of CLL cell decline during ibrutinib treatment slows down over time and converges to a plateau. In accordance with data, it is assumed that no meaningful amount of cell proliferation and homing occurs during therapy. (B) Two examples of the treatment responses to ibrutinib. Circles show measured ALC in the blood, and the line is the best model fit to the data. The top graph is a typical patient with "slow" disease clearance, with a pronounced lymphocytosis phase that lasts for about 3 weeks. The bottom graph is a typical "fast" responder, with limited lymphocytosis that only lasts for a few days and a subsequent rapid decline. (C) Distribution of tissue death rates of tumor cells among patients, separated according to M-CLL (blue) and U-CLL (red). Each symbol represents a patient. The death rate is expressed both as the average life span of CLL cells in a patient (left), and as the percentage of CLL cells that die per day (right). (D) Average tissue decline dynamics among patients with M-CLL (blue) and U-CLL (red), as predicted by the parameterized mathematical model. The computer simulation of the model was run for individual patients, and the predicted tissue sizes were averaged and plotted every 30 days. The tissue size before start of treatment is given by 100 , and the other sizes are scaled accordingly. For the purpose of comparison to previous studies, we plotted the predicted $2 \mathrm{D}$ tissue size, which scales with the number of cells to the power of $2 / 3$. Note that this assumes complete correspondence between tissue size shrinkage and the decline of cell numbers in tissue, which should be reasonably accurate for large tissue sizes. Deviations are expected at smaller tissue sizes.

$0.39 \%$ per day before therapy to $0.05 \%$ per day on ibrutinib therapy (Figure $2 \mathrm{~F}$ ). This key result is evident by the remarkable stability in the fraction of ${ }^{2} \mathrm{H}$-labeled CLL cells detected in the blood after ibrutinib administration when ${ }^{2} \mathrm{H}_{2} \mathrm{O}$ had washed out of the body water pool (Figure $2 \mathrm{C}$ ). Dilution of previously divided, ${ }^{2} \mathrm{H}$-labeled CLL cells by unlabeled CLL cells would occur if there was ongoing birth of new CLL cells, as demonstrated in previous cohorts of untreated CLL patients after washout of ${ }^{2} \mathrm{H}_{2} \mathrm{O}$ from the body water pool (19). 
Prior studies in patients with CLL using the ${ }^{2} \mathrm{H}_{2} \mathrm{O}$-labeling approach established that CLL cells proliferate at rates ranging from approximately $0.1 \%$ to approximately $2.0 \%$ of the clone per day (19). Here, we measured a median CLL cell birth rate before ibrutinib therapy of $0.39 \% \pm 0.21 \%$, which is similar to these prior data.

Our findings further document that ibrutinib therapy promotes the death of CLL cells. The CLL cell death rate in the blood increased from $0.18 \%$ per day before therapy to $1.5 \%$ per day after the start of ibrutinib (Figure 2F). Because the leukemic cells could not leave the circulation because of the inability to follow a CXCL12 gradient due to inhibition of CXCR4 action by ibrutinib, our measurements establish inherent death rates for individual CLL clones. Moreover, our analysis of the tissue compartment dynamics revealed that the estimated cell death rate in the tissues during ibrutinib therapy was even higher than in the blood and that tissue CLL cell death rates correlated with genetic risk factors and clinical outcome. Interestingly, higher-risk patients with unmutated IGHV (U-CLL) displayed larger tissue cell death rates and responded more promptly to ibrutinib therapy. Accordingly, U-CLL patients had more rapid clearance of CLL cells from the blood (Figure 3B), bone marrow (Figure 3F), and secondary lymphoid tissue sites (Figure 5). These observations are in accordance with those of a previous report about more delayed peripheral blood clearance in M-CLL patients undergoing ibrutinib therapy and point toward lesser or higher dependencies of M-CLL versus U-CLL cells, respectively, on functional BTK and presumably on survival and growth signals delivered via the BCR and other receptors. This conclusion is consistent with higher BCR signaling capacity and dependency in U-CLL $(22,23)$.

Regarding the increased CLL cell death rates on ibrutinib therapy, we cannot determine from our study design whether this is a direct or indirect effect of the drug. Clinically, ibrutinib has not been commonly associated with symptoms or signs of tumor lysis, including major increases in uric acid or LDH levels, as observed with fast-acting conventional chemotherapy agents or BCL2 antagonists. These observations argue against major direct cytotoxicity induced by ibrutinib. In addition, the significant transient increase in CLL cell counts in the peripheral blood (1) can be reversed by treatment interruption (8). These characteristic clinical findings led to the proposal that displacement of leukemia cells from their supportive microenvironment, followed by leukemia cell death due to "death by neglect," is a central mechanism of action of ibrutinib and other kinase inhibitors, such as the PI3 kinase $\delta$ inhibitor idelalisib (12). At the tissue level, death could result from detaching CLL cells from their supportive tissue environment, a form of programmed cell death resembling anoikis (24), which aborts short-range survival signals through the $\operatorname{BCR}(15,25)$ and other receptors, such as $\operatorname{CXCR} 4(15,16)$ and integrins $(26)$. This is followed by clearance of apoptotic cells by local phagocytic cells, as seen in the thymus and other areas in which clonal abortion occurs (27).

Our data further confirm that ibrutinib treatment leads to CLL cell redistribution from tissues into the blood, putting to rest the initial concern that the early transient increase in blood CLL cell counts is due to a burst in CLL cell proliferation. Redistribution is more prominent in patients with M-CLL and in previously treated patients and less prominent or absent in untreated patients with U-CLL, as shown here. One explanation for this heterogeneity points again to differential drug sensitivity in terms of CLL cell survival between U-CLL and M-CLL, as discussed above. While M-CLL cells are less BCR dependent (22) and therefore can survive BTK inhibition for longer periods of time, more robust subclones may better survive the transition from tissues into the blood, where they can survive for extended periods of time (11). The majority of U-CLL cells, especially when previously untreated, are more sensitive to and do not survive BTK inhibition, even for short periods of time, and therefore one might not detect a major or sustained elevation in peripheral blood CLL cells after the start of ibrutinib therapy.

In summary, this study reports several observations that are relevant to the therapeutic use of a targeted therapy, the BTK inhibitor ibrutinib, in CLL. We show for the first time to our knowledge directly in patients with CLL that ibrutinib immediately and persistently abrogates the proliferation of leukemia cells and at the same time permits increased rates of their death. The findings also establish inherent death rates for individual CLL clones. Moreover, we have proven in patients that the early lymphocytosis observed after initiation of ibrutinib treatment is not due to CLL cell proliferation but reflects redistribution of cells, as has been assumed by clinical observation, and that patients with U-CLL exhibit higher rates of tissue cell death and less redistribution after initiation of therapy as well as better clinical outcomes on ibrutinib, as has been gleaned from clinical trials (11). Hence, ibrutinib very effectively directly blocks the proliferation of CLL cells and indirectly promotes their death, thereby clearing a major fraction of the disease burden within the first weeks of therapy. 


\section{Table 1. Patient characteristics.}

\begin{tabular}{|c|c|}
\hline Characteristics & $n(\%)$ or median (range) \\
\hline Age (yr) & $64(48-78)$ \\
\hline wbc $\left(\times 10^{9} / I\right)$ & $98.2(34.2-258.3)$ \\
\hline Hemoglobin (g/dl) & $12.2(7.0-13.8)$ \\
\hline Platelets $\left(\times 10^{9} / \mathrm{I}\right)$ & $111.5(68-254)$ \\
\hline$\beta 2$-microglobulin ( $\mathrm{mg} / \mathrm{l})$ & $2.8(1.6-7.4)$ \\
\hline \multicolumn{2}{|l|}{ Sex } \\
\hline Male & $19(63.3)$ \\
\hline Female & $11(36.7)$ \\
\hline \multicolumn{2}{|l|}{ Rai stage } \\
\hline 0 & $1(3.3)$ \\
\hline I & $9(30.0)$ \\
\hline II & $6(20.0)$ \\
\hline III & $4(13.3)$ \\
\hline IV & $10(33.3)$ \\
\hline \multicolumn{2}{|l|}{ Cytogenetics } \\
\hline $13 Q$ deletion & $11(36.7)$ \\
\hline Diploid & $6(20)$ \\
\hline Trisomy 12 & $6(20)$ \\
\hline $11 Q$ deletion & $4(13.3)$ \\
\hline 17P deletion & $3(10)$ \\
\hline \multicolumn{2}{|l|}{ IGVH mutational status } \\
\hline Mutated & $11(36.7)$ \\
\hline Unmutated & $17(56.7)$ \\
\hline \multicolumn{2}{|l|}{ ZAP-70 (IHC) } \\
\hline Negative & $15(50)$ \\
\hline Positive & $15(50)$ \\
\hline \multicolumn{2}{|l|}{ CD38 } \\
\hline Negative & $22(73.3)$ \\
\hline Positive & $8(26.7)$ \\
\hline
\end{tabular}

\section{Methods}

Patients and treatment. Thirty previously untreated CLL/SLL patients were enrolled between December 2012 and June 2013 at MD Anderson Cancer Center. Patients had active CLL with a need for treatment per iwCLL guidelines (28). The reasons for treatment initiation for each patient are listed in Supplemental Table 1; patients with a lymphocyte doubling time of $<3$ months or other clinical or laboratory signs of rapidly progressive disease were excluded. All patients had adequate hematopoietic (platelets $>50,000 / \mu 1$, ANC $>750 / \mu 1$ ) and organ functions. Patient characteristics are summarized in Table 1 . Patients drank $50 \mathrm{ml}$ of ${ }^{2} \mathrm{H}_{2} \mathrm{O}(70 \%$ enriched; Cambridge Isotope Laboratories Inc.) 3 times a day for 5 days, followed by $60 \mathrm{ml}$ daily for a total of 4 weeks during the "labeling phase" (Figure 1). This schedule led to approximately $1.0 \%$ to $2.0 \%$ body ${ }^{2} \mathrm{H}_{2} \mathrm{O}$ enrichment that was reached and stably maintained during the labeling period (Figure 2A). These levels of ${ }^{2} \mathrm{H}_{2} \mathrm{O}$ enrichment have no known adverse effects in humans (29). Patients returned at scheduled intervals during and following the labeling period to donate blood for cell and serum analyses. In addition, all patients kept a daily log of ${ }^{2} \mathrm{H}_{2} \mathrm{O}$ intake. After a 6- to 12-week "washout phase" following discontinuation of ${ }^{2} \mathrm{H}_{2} \mathrm{O}$ intake, during which time body ${ }^{2} \mathrm{H}_{2} \mathrm{O}$ enrichments fell to near 0 values (Figure 2A), patients started once-daily ibrutinib at a dose of $420 \mathrm{mg}$ continuously on 28-day cycles (see Figure 1). Thus, during the ${ }^{2} \mathrm{H}_{2} \mathrm{O}$ intake "labeling phase," all proliferating cells would be tagged with ${ }^{2} \mathrm{H}$ in the deoxyribose moiety of their newly replicated DNA and during the post-washout "delabeling" phase, proliferating cells would not be tagged and would dilute any previously tagged cells. Study objectives were to determine the effect of ibrutinib therapy on CLL cell proliferation rates, death rates, and trafficking patterns. Treatment with ibrutinib continued until disease progression or toxicities occurred or until complications precluded further therapy.

Cell separation, isotopic enrichment in CLL cell DNA, and calculation of CLL birth and death rates. In vivo labeling of proliferating CLL cells was accomplished by drinking small volumes of ${ }^{2} \mathrm{H}_{2} \mathrm{O}$ daily over 4 weeks, allowing metabolic incorporation of ${ }^{2} \mathrm{H}$ into the DNA of cells that divided during the period of ${ }^{2} \mathrm{H}_{2} \mathrm{O}$ exposure. CLL cells were purified from serial blood specimens and analyzed for isotope enrichment in cellular DNA by gas chromatography/pyrolysis/isotope ratio-mass spectrometry, as described previously (30). CLL cell proliferation was estimated in two complementary ways: first, during the period of ${ }^{2} \mathrm{H}_{2} \mathrm{O}$ exposure in vivo, based on the rate of ${ }^{2} \mathrm{H}$ incorporation into DNA of CLL cells; and, second, during the period after ${ }^{2} \mathrm{H}_{2} \mathrm{O}$ had washed out of the body water pool (Figure 2A), based on the dilution of previously ${ }^{2} \mathrm{H}$-labeled CLL cells by newly divided unlabeled cells. Death rates $\left(k_{d}\right)$ after ibrutinib treatment were calculated as the sum of the measured birth rate plus the net exponential loss rate in blood ALC, while death rates before therapy were calculated as the difference between the measured birth rate and the net exponential growth rate in blood ALC. Further methodological details are provided in the supplement in the section called Calculation of fractional birth and death rates from CLL labeling data and CLL cell counts.

Tissue death rates. The volume of lymphoid tissues and the spleen was quantified by CT scans prior to therapy and during ibrutinib therapy in order to quantify changes in tissue CLL disease burden, as described previously (21). Briefly, volumes of lymph nodes and spleen were calculated using CT-based volumetric analyses with 3-dimensional reconstruction of each lesion, measuring a combination of axial contours and craniocaudal diameters. Examples of target lesions and their respective volumes before and during therapy are depicted in Figure 4. Tissue CLL death rates were estimated based on kinetic changes in tissue volumes and ALCs, using methodology established earlier by us (21). Briefly, changes in lymph node and spleen volumes were calculated for each patient, based on CT scans obtained prior to therapy and at 3 months after initiation of treatment. These volume changes were translated into numbers of affected tissue CLL cells per patient and set into relation with dynamic changes in serial blood lymphocyte counts, using average CLL cell volumes and individual blood volumes.

Statistics. Statistical analyses were conducted using GraphPad Prism version 6.00 for Windows (GraphPad Software). For all analyses, significance was set at $P<0.05$ (2 tailed). Descriptive statistical analyses, 
including histograms or box plots, proportions, ranges, means, and standard deviations were calculated. Fisher's exact test and Wilcoxon rank test were used in univariate analyses of categorical and continuous variables, respectively. Data analyses were performed based on the intent to treat. Survival or times to progression functions were estimated using the Kaplan-Meier method. Toxicity was reported by type, frequency, and severity. Worst toxicity grades per patient were tabulated for selected adverse events and laboratory measurements. The Department of Biostatistics at MD Anderson Cancer Center provided and maintained a website ("Clinical Trial Conduct," https://biostatistics.mdanderson.org/ClinicalTrialConduct/) for enrolling patients on this study and evaluating the efficacy monitoring rules described above.

Study approval. This pilot study was developed by the investigators in collaboration with Pharmacyclics, was approved by The University of Texas MD Anderson Cancer Center Institutional Review Board, and is registered at clinicaltrials.gov (NCT01752426). Informed consent was obtained in accordance with institutional guidelines and the Declaration of Helsinki. The study was conducted in accordance with the Declaration of Helsinki and was sponsored by Pharmacyclics, an AbbVie Company.

\section{Author contributions}

KWL, MS, MKH, SMT, CLE, SSC, and XJY performed cell purification, DNA extraction, and gas chromatography/mass spectometry analyses, and collected and analyzed the data with XH. DW performed mathematical data modeling. AMA and NG performed CT volumetric analyses and designed Figure 4. MJK, AF, HK, WGW, and SO provided patient samples and reviewed the manuscript. JAB and NC designed the research, supervised the study, analyzed the data, and wrote the paper with DW, SSC, $\mathrm{MKH}$, and KWL. All investigators and their research teams collected the data. The investigators confirmed the accuracy of the data and compiled these for analysis. All authors had full access to the data and were involved in data interpretation as well as with the revision and final approval of the manuscript. All authors vouched for accuracy of the reported data, confirmed adherence to the protocol, and made the decision to submit the data for publication.

\section{Acknowledgments}

We thank the patients who participated in this trial and their families as well as the research nurses and study coordinators at MD Anderson who helped to carry it out. The study was supported by a Cancer Center Support Grant (National Cancer Institute grant P30 CA016672), an NIH grant (CA081554) from the National Cancer Institute (to NC), MD Anderson's Moon Shots Program in CLL, and Pharmacyclics, an AbbVie Company. JAB is a Leukemia \& Lymphoma Society Scholar in Clinical Research.

Address correspondence to: Jan A. Burger, Department of Leukemia, Unit 428, The University of Texas MD Anderson Cancer Center, 1400 Holcombe Boulevard, Houston, Texas 77030, USA. Phone: 713.563.1487; E-mail: jaburger@mdanderson.org. Or to: Nicholas Chiorazzi, The Feinstein Institute for Medical Research, Northwell Health, 350 Community Drive, Manhasset, New York 11030, USA. Phone: 516.562.1090; E-mail: NChizzi@NSHS.edu.

1. Byrd JC, et al. Targeting BTK with ibrutinib in relapsed chronic lymphocytic leukemia. N Engl J Med. 2013;369(1):32-42.

2. Wiestner A. BCR pathway inhibition as therapy for chronic lymphocytic leukemia and lymphoplasmacytic lymphoma. Hematology Am Soc Hematol Educ Program. 2014;2014(1):125-134.

3. Furman RR, et al. Idelalisib and rituximab in relapsed chronic lymphocytic leukemia. N Engl J Med. 2014;370(11):997-1007.

4. Byrd JC, et al. Ibrutinib versus ofatumumab in previously treated chronic lymphoid leukemia. $N$ Engl J Med. 2014;371(3):213-223.

5. Goede V, et al. Obinutuzumab plus chlorambucil in patients with CLL and coexisting conditions. N Engl J Med. 2014;370(12):1101-1110.

6. Honigberg LA, et al. The Bruton tyrosine kinase inhibitor PCI-32765 blocks B-cell activation and is efficacious in models of autoimmune disease and B-cell malignancy. Proc Natl Acad Sci U S A. 2010;107(29):13075-13080.

7. Burger JA, Buggy JJ. Bruton tyrosine kinase inhibitor ibrutinib (PCI-32765). Leuk Lymphoma. 2013;54(11):2385-2391.

8. Advani RH, et al. Bruton tyrosine kinase inhibitor ibrutinib (PCI-32765) has significant activity in patients with relapsed/refractory B-cell malignancies. J Clin Oncol. 2013;31(1):88-94.

9. Burger JA, et al. Ibrutinib as initial therapy for patients with chronic lymphocytic leukemia. N Engl J Med. 2015;373(25):2425-2437.

10. Byrd JC, et al. Three-year follow-up of treatment-naïve and previously treated patients with CLL and SLL receiving single-agent ibrutinib. Blood. 2015;125(16):2497-2506.

11. Woyach JA, et al. Prolonged lymphocytosis during ibrutinib therapy is associated with distinct molecular characteristics and does not indicate a suboptimal response to therapy. Blood. 2014;123(12):1810-1817. 
12. Burger JA, Montserrat E. Coming full circle: 70 years of chronic lymphocytic leukemia cell redistribution, from glucocorticoids to inhibitors of B-cell receptor signaling. Blood. 2013;121(9):1501-1509.

13. Cheson BD, et al. Novel targeted agents and the need to refine clinical end points in chronic lymphocytic leukemia. J Clin Oncol. 2012;30(23):2820-2822.

14. Herman SE, et al. Bruton tyrosine kinase represents a promising therapeutic target for treatment of chronic lymphocytic leukemia and is effectively targeted by PCI-32765. Blood. 2011;117(23):6287-6296.

15. Ponader S, et al. The Bruton tyrosine kinase inhibitor PCI-32765 thwarts chronic lymphocytic leukemia cell survival and tissue homing in vitro and in vivo. Blood. 2012;119(5):1182-1189.

16. de Rooij MF, et al. The clinically active BTK inhibitor PCI-32765 targets B-cell receptor- and chemokine-controlled adhesion and migration in chronic lymphocytic leukemia. Blood. 2012;119(11):2590-2594.

17. Herman SE, et al. Modeling tumor-host interactions of chronic lymphocytic leukemia in xenografted mice to study tumor biology and evaluate targeted therapy. Leukemia. 2013;27(12):2311-2321.

18. Chen SS, et al. BTK inhibition results in impaired CXCR4 chemokine receptor surface expression, signaling and function in chronic lymphocytic leukemia. Leukemia. 2016;30(4):833-843.

19. Messmer BT, et al. In vivo measurements document the dynamic cellular kinetics of chronic lymphocytic leukemia B cells. $J$ Clin Invest. 2005;115(3):755-764.

20. Murphy EJ, et al. Kinetic measurement of leukemia-cell proliferation rate by deuterium labeling predicts time to initial treatment of patients with chronic lymphocytic leukemia. Blood. 2014;124 (21):829.

21. Wodarz D, et al. Kinetics of CLL cells in tissues and blood during therapy with the BTK inhibitor ibrutinib. Blood. 2014;123(26):4132-4135.

22. Chen L, et al. Expression of ZAP-70 is associated with increased B-cell receptor signaling in chronic lymphocytic leukemia. Blood. 2002;100(13):4609-4614.

23. Burger JA, Chiorazzi N. B cell receptor signaling in chronic lymphocytic leukemia. Trends Immunol. 2013;34(12):592-601.

24. Frisch SM, Screaton RA. Anoikis mechanisms. Curr Opin Cell Biol. 2001;13(5):555-562.

25. Cheng S, et al. BTK inhibition targets in vivo CLL proliferation through its effects on B-cell receptor signaling activity. Leukemia 2014;28(3):649-657.

26. de la Fuente MT, Casanova B, Garcia-Gila M, Silva A, Garcia-Pardo A. Fibronectin interaction with alpha4beta1 integrin prevents apoptosis in B cell chronic lymphocytic leukemia: correlation with Bcl-2 and Bax. Leukemia. 1999;13(2):266-274.

27. Henson PM, Hume DA. Apoptotic cell removal in development and tissue homeostasis. Trends Immunol. 2006;27(5):244-250.

28. Hallek M, et al. Guidelines for the diagnosis and treatment of chronic lymphocytic leukemia: a report from the International Workshop on Chronic Lymphocytic Leukemia updating the National Cancer Institute-Working Group 1996 guidelines. Blood. 2008;111(12):5446-5456.

29. Busch R, Neese RA, Awada M, Hayes GM, Hellerstein MK. Measurement of cell proliferation by heavy water labeling. Nat Protoc. 2007;2(12):3045-3057.

30. Voogt JN, Awada M, Murphy EJ, Hayes GM, Busch R, Hellerstein MK. Measurement of very low rates of cell proliferation by heavy water labeling of DNA and gas chromatography/pyrolysis/isotope ratio-mass spectrometric analysis. Nat Protoc. 2007;2(12):3058-3062. 